\title{
Gas Jet Coal-Breaking Behavior: An Elliptical Crushing Theoretical Model
}

\author{
Gongda Wang, ${ }^{1,2}$ Yuanyuan Wang $\mathbb{D}^{1,2}$ Xin Yang, ${ }^{1,2}$ and Xin Song ${ }^{1,2}$ \\ ${ }^{1}$ School of Civil and Resources Engineering, University of Science and Technology Beijing, Beijing 100083, China \\ ${ }^{2}$ State Key Laboratory of the Ministry of Education of China for High-Efficiency Mining and Safety of Metal Mines, University of \\ Science and Technology Beijing, Beijing 100083, China
}

Correspondence should be addressed to Yuanyuan Wang; g20198146@xs.ustb.edu.cn

Received 26 July 2021; Revised 20 August 2021; Accepted 21 September 2021; Published 12 October 2021

Academic Editor: Afshin Davarpanah

Copyright (c) 2021 Gongda Wang et al. This is an open access article distributed under the Creative Commons Attribution License, which permits unrestricted use, distribution, and reproduction in any medium, provided the original work is properly cited.

Coalbed methane $(\mathrm{CBM})$ is a source of clean energy and has been recovered in past decades all over the world. Gas dynamic disaster is the primary disaster in outburst coal, and methane drainage plays a key role in eliminating this danger. As an efficient technology, a gas jet is widely used in CBM development and methane drainage. In this work, the full impinging process of coal and rock fracturing by a supersonic gas jet was studied. To understand how jet parameters affect coal and rock fracturing results, an elliptical crushing theoretical model was proposed. In addition, a laboratory experiment was designed to examine the proposed model, and four key parameters affecting the fracturing results were studied. The results show that different from the monotonic variation of theoretical values, there is a turning point in the variation of experimental values under some parameters. Considering the influence of the depth and radius of the erosion pit, the rock-breaking effect is better when the nozzle size is $2.75 \mathrm{Ma}$. The optimal target distance is $30 \mathrm{~mm}$, and the impact pressure of a gas jet should be continuously increased in order to achieve certain rock-breaking effects under the impact of the jet.

\section{Introduction}

Coalbed methane (CBM) is a source of clean energy and has been recovered in past decades all over the world [1-4]. In terms of coal mining, methane is the primary cause of mine disasters especially for outburst mines [5-7]. Therefore, methane drainage plays a key role in eliminating gas outbursts. To enhance CBM recovery or drainage efficiency, water jet technology has been proposed and has been proven effective [8-12]. However, in the low permeability soft coal seams, the use of water jet technology frequently leads to the collapse of a borehole and the inhabitation of the gas desorption [13-16]. Due to the fact that the use of a gas jet for the fracture of coal can effectively avoid these problems, more and more attention has been paid to gas jet technology.

Gas jet technology is widely used in the fields of glass cutting, hole drilling, and cooling [17-19]. Komori et al. [20] proposed a supersonic gas jet technology based on the Laval nozzle. Frendi and Brown [21] studied the flow structures from a supersonic impinging jet and indicated a strong interaction between the acoustic waves emanating from the impingement plate and the jet plume. Due to the high impact force of a high-pressure gas jet, it has been extended to the fracture of coal and rock in recent years. Kutter and Fairhurst [22] carried out the research of high-pressure gas punching fracture earlier, and they proposed that the pressure distribution along the normal direction of a rock fracture can be produced in the rock when high-pressure gas impacts rock materials. Hagan [23] proposed the "gas wedge effect" by comparing the action form of high-pressure gas with the mechanism of a water jet. On the basis of previous studies, Nilson et al. [24] established a model of highpressure gas punching fracture. Eslamian et al. [25] studied the crushing effect of a supersonic gas jet on a cylindrical brittle gypsum deposit. It was found that both jet angle and sediment hardness can affect the crushing efficiency. Liu et al. [26-29] proposed a high-pressure abrasive gas jet technology for coal breakage and systematically investigated the stress wave effect, the wear mechanism of abrasive air jet erosion, and the effects of nozzle pressure ratios on the 
pulsation frequency of air jets. Ranjith et al. [30] studied the effect of mass flow on the abrasive acceleration of gas jets. They found that pressure is also important. When the mass flow increases, the abrasive acceleration distance and time decrease.

Previous studies mainly focused on optimizing the nozzle structure and jet parameters to improve the efficiency of fracturing coal and rock [25, 28]. However, highpressure gas jet coal breaking is a continuous and comprehensive process including the transportation of highpressure gas in the pipeline, the free development of highpressure gas in the air medium, and the final coal breaking. Researchers mainly focused on the stage of final coal breaking by jet punching, and the study on the whole continuous process of gas jet impinging coal and rock is relatively rare. Moreover, as for the research on the damage characteristics of a high-pressure gas jet, there is lack of the corresponding criterion for coal breaking by a gas jet. Therefore, in this work, the full process of a high-pressure gas jet was systematically considered, and a mathematical model was proposed. The supersonic air jet impinging laboratory experiment was designed to examine the proposed model and four key parameters affecting the fracturing results were tested, including jet pressure, target distance, nozzle size, and strength of coal and rock. This study is intended to enrich the theories of gas jet coal breaking, providing some guidance for the development of CBM and the prevention of mine gas disasters.

\section{Mathematical Model of the Full Impinging Progress of a Supersonic Gas Jet}

As for a full impinging system, considering the risk of highpressure gas, the air compressor and high-pressure gas storage tank were placed in an underground chamber, and then the high-pressure air reaches the nozzle through the pipeline to fracture the coal and rock. The system is shown in Figure 1. The introduction of the mathematical model of the full impinging progress of a supersonic gas jet follows.

2.1. Flow Model of High-Pressure Gas in a Pipeline. The viscosity of gas shall be considered in the mechanical energy balance formula of pipeline calculation gas, that is, there shall be resistance loss term $h_{f}$ in the formula:

$$
g z_{1}+\frac{u_{1}^{2}}{2}+\int_{p_{1}}^{p_{2}} \frac{d p}{\rho}=g z_{2}+\frac{u_{2}^{2}}{2}+h_{f} .
$$

When the gas flows in the pipeline, the volume flow rate and average flow rate change along the length of the pipe, and the resistance loss per unit length of the pipe must also change along the length of the pipe. Formula (1) above is changed to the differential form below:

$$
g d z+d \frac{u^{2}}{2}+v d p+\lambda \frac{(d l)}{d} \times \frac{u^{2}}{2}=0
$$

where $v=(1 / \rho)=(R T / M p)$ is the specific volume of gas $\left(\mathrm{m}^{3} / \mathrm{kg}\right), L$ is the pipe length $(\mathrm{m}), d$ is the pipe diameter (m), friction coefficient $\lambda$ is a function of $\operatorname{Re}$ and $\varepsilon / d$, and we have

$$
\operatorname{Re}=\frac{d u \rho}{\mu}=\frac{d G}{\mu} .
$$

In the case of equal pipe diameter transportation, since the mass flow rate $G$ is a constant along the pipe length, $\mathrm{Re}$ is only related to the temperature of the gas. Therefore, $\lambda$ can be regarded as a constant along the length of the tube for the flow process with little change in isothermal flow or temperature:

$$
G=\frac{q_{m}}{A}
$$

where $q_{m}$ is the mass flow $(\mathrm{kg} / \mathrm{S}), A$ is the cross-sectional area of the pipe $\left(\mathrm{m}^{2}\right)$ :

$$
u=\frac{G}{\rho}=G v
$$

For isothermal flow, $p^{*} v$ is equal to the constant. And substituting equation (5) into equation (2), we can get

$$
G^{2} \ln \frac{p_{1}}{p_{2}}+\frac{p_{2}^{2}-p_{1}^{2}}{(2 R T / M)}+\lambda \frac{l}{2 d} G^{2}=0 .
$$

If the pressure drop $\Delta p$ in the tube is very small, the kinetic energy difference of the first term in equation (6) can be ignored. In this case, equation (6) is the special form of the energy equation of the incompressible fluid to the horizontal tube. For the transportation of high-pressure gas, ( $\left.p_{1}-p_{2}\right) / p_{2}$ is smaller and can be treated as an incompressible fluid. After finishing formula (6), we can conclude the following:

$$
\left(p_{2}^{2}-p_{1}^{2}\right) \frac{M}{2 R T}+\lambda \frac{l}{2 d} G^{2}=0
$$

Under the condition of known $T$, pipeline parameters, pipeline outlet pressure $p_{2}$ (equal to nozzle inlet pressure), and the pressure $p_{1}$ at the pipeline inlet can be obtained. Therefore, $p_{2}$ can be achieved by adjusting $p_{1}$ to supply high-pressure gas remotely.

2.2. Mathematical Model of Continuous Process and Criterion of Coal Breaking by Jet. The jet is accelerated through the nozzle, improving the transition from subsonic to supersonic in the throat. Then, the gas jet is ejected from the nozzle into the environmental medium. Finally, the supersonic jet impinges on the coal wall and forms an erosion pit on the target surface. This process can be divided into three parts: the acceleration of the gas jet, the development of the gas jet, and the impact of the gas jet.

2.2.1. Mathematical Model of Acceleration Stage. The nozzle is the key factor affecting jet velocity. The straight or conical 


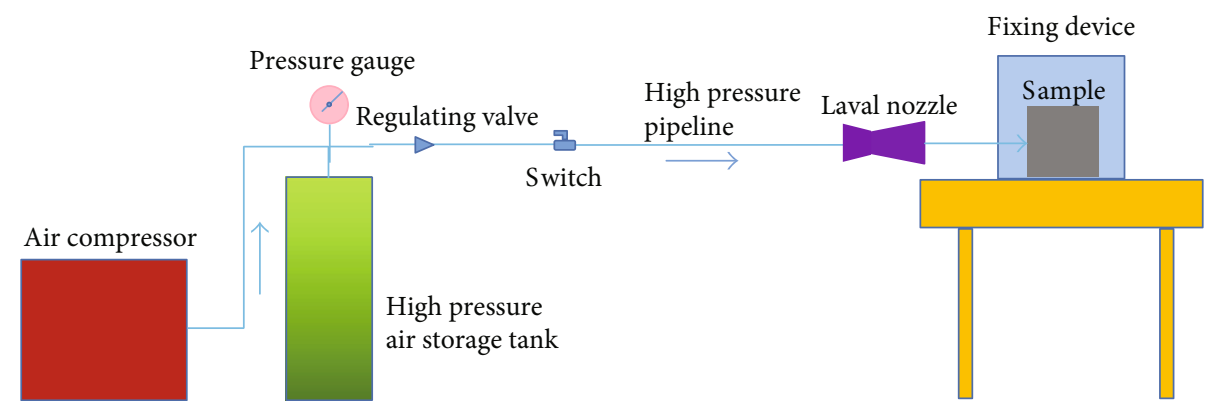

FIGURE 1: Schematic diagram of full impinging system.

convergent nozzles are generally used in traditional gas jet technology, and the highest speed that a gas jet can reach is sonic. In order to improve the working efficiency, the common conical contraction nozzle is often used in engineering applications. After the high-pressure gas flows through the contraction nozzle, the maximum can only reach one Mach number, which is equivalent to the sound velocity flow of local air. Under the same external parameters, the Laval nozzle can achieve speeds several times the sound velocity. The nozzle is usually composed of a convergence section, a throat part, and an expansion section. Its structure is shown in Figure 2.

The aerodynamics theory indicates that when the highpressure gas flows through the nozzle with known parameters, the gas state after acceleration depends on the nozzle parameters, inlet air pressure, temperature, and outlet external back pressure. When the ratio of a nozzle area to the throat area is determined, the Mach number after the acceleration can be obtained from the following formula [31]:

$$
\frac{S}{S_{e}}=\frac{1}{\mathrm{Ma}}\left[\left(1+\frac{k-1}{2} \mathrm{Ma}^{2}\right) \times\left(\frac{2}{k+1}\right)\right]^{(k+1 / 2(k-1))} .
$$

In order to simplify the complexity of the problem, an imaginary reference state is introduced in the study of aerodynamics. The state where the velocity of air decreases to zero after the absolute energy isentropic process from a real state is called the stagnation state corresponding to the real state. In the stagnation state, it is assumed that the flow process of high-pressure gas in the nozzle is a one-dimensional steady isentropic adiabatic flow. There is no heat and work exchange between the gas in the flow and the outside world. No flow is added or drawn out, and the friction between the high-pressure gas and the nozzle wall is ignored. For the Laval nozzle, the supersonic flow in the nozzle can be expressed by the flow parameters in the stagnation state:

$$
\left\{\begin{array}{l}
\frac{P^{*}}{P}=\left(1+\frac{k-1}{2} \mathrm{Ma}^{2}\right)^{(k / k-1)}, \\
\frac{\rho^{*}}{\rho}=\left(1+\frac{k-1}{2} \mathrm{Ma}^{2}\right)^{(k / k-1)}, \\
\frac{T^{*}}{T}=1+\frac{k-1}{2} \mathrm{Ma}^{2},
\end{array}\right.
$$

where $P$ is the gas pressure, $P^{*}$ is the gas pressure under stagnation state, $\rho^{*}$ is the gas density under the stagnation state, $\rho$ is the gas density, $T$ is the gas thermodynamic temperature, $T^{*}$ is the gas thermodynamic temperature under the stagnation state, and $\mathrm{Ma}$ is the Mach number, which means

$$
\mathrm{Ma}=\frac{V}{a},
$$

where $a$ is the corresponding local air sound velocity in this state, which can be obtained from $a=\sqrt{k R T} ; R$ is the gas constant, and the value for air is $287.06 \mathrm{~J} /(\mathrm{kg} \cdot \mathrm{K}) ; T$ is the gas thermodynamic temperature.

According to formulas (8) to (10), the flow velocity at the nozzle exit section can be obtained from formula (11) combining the total pressure at the nozzle inlet, the total temperature at the nozzle outlet, and the external back pressure at the nozzle outlet:

$$
U_{0}=\left\{\begin{array}{l}
\sqrt{\frac{2}{k-1}\left[\left(\frac{P^{*}}{P}\right)^{(k-1 / k)}-1\right]} \cdot \sqrt{k R T}, \quad \mathrm{Ma}<\mathrm{Ma}_{\max }, \\
\mathrm{Ma}_{\max } \cdot \sqrt{k R T}, \quad \mathrm{Ma}=\mathrm{Ma}_{\max },
\end{array}\right.
$$

where $\mathrm{Ma}_{\max }$ is the maximum Mach number that can be reached at the outlet under the given nozzle parameters.

2.2.2. Mathematical Model of Development Stage. The gas jet is ejected from the nozzle into the environmental medium until it strikes a certain wall. The development characteristics of the jet can be divided into three typical zones, namely, the free jet zone, the impinging zone, and the wall jet zone $[32,33]$. The zoning diagram is shown in Figure 3.

In the free jet zone, the shear driving interactions exist between the outer boundary of the jet and the surrounding air medium. The existence of shear stress would lead to the generation of turbulence, which causes the attenuation of the jet velocity in the axial direction. Meanwhile, it would aggravate the entrainment of the air fluid around the jet, resulting in the increase of the total jet flow and the continuous expansion of the jet cross-section along the radial direction. However, the static pressure of the 


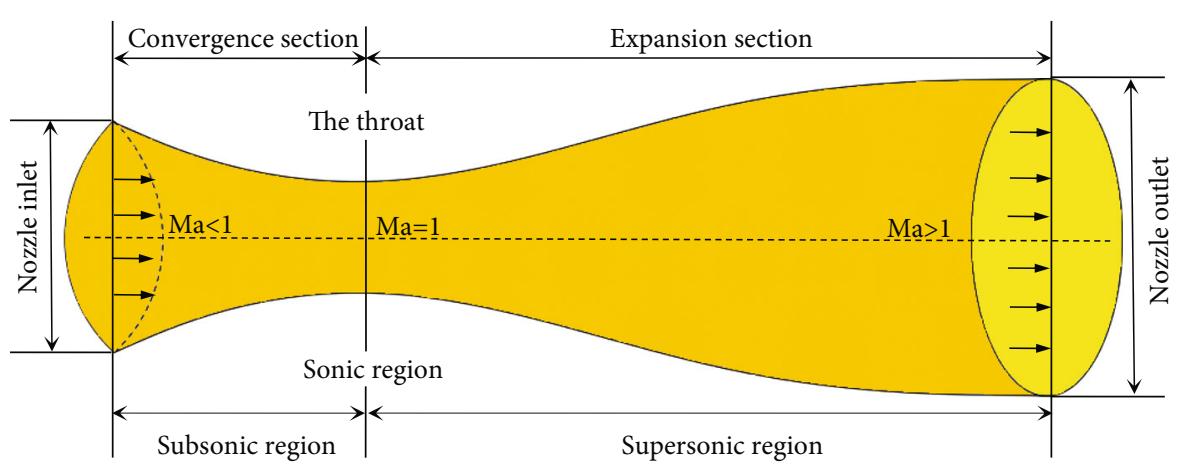

Figure 2: Schematic diagram of a scaled nozzle structure.

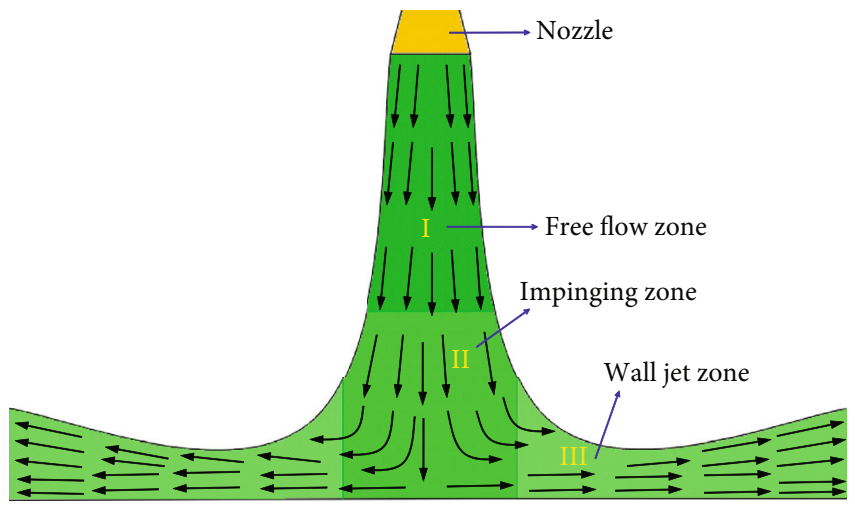

FIGURE 3: Schematic diagram of gas jet development zones.

jet on the wall begins to rise, showing a large pressure gradient and forcing the jet streamline to produce rapid bending. The jet flow changes from the original axial flow to the near wall flow, which is characterized by the existence of a stagnation point and the radial bending of the jet streamline. In the wall jet zone, the jet generally presents along the diameter. Due to the shear interaction between the wall and the air medium, the jet velocity begins to decay to zero after a long distance from the stagnation point.

The influence factors of the development stage of the gas jet include nozzle radius $b_{0}$, nozzle plate distance $H$, incidence angle $\theta$, and jet initial velocity $U_{0}$. In order to facilitate the analysis, a rectangular coordinate system $(x, y)$ is established in the jet flow field to analyze the free jet zone and the impingement zone, and the coordinate system $\left(x_{1}, y_{1}\right)$ is used to analyze the impinging zone and the wall jet zone, as shown in Figure 4.

The axial pressure of a jet is defined as $P_{m}$. The pressure at the stagnation point and the wall pressure are $P_{S}$ and $P_{W}$, respectively. $U_{m}$ represents the jet velocity, and $U_{m 1}$ is the maximum value of $U_{m}$ in the $x_{1}$ direction of any crosssection. $b_{u}$ represents the radial length dimension of the fluid cross-section velocity of $1 / 2 U_{m}$ in the free jet section. According to the analysis, starting from the stagnation point, $U_{m 1}$ increases with the increase of $x_{1}$ until the maximum value. Then, the velocity decreases and tends to be zero at $U_{m 1}$ far away from the jet axis due to the fact that the turbu- lence is mixed with the surrounding air medium. In the free jet zone, the axial velocity $U_{m f}$ and radial length $b_{f}$ can be expressed as follows:

$$
U_{m f}, b_{f}=f_{1,2}\left(M_{0}, \rho, x\right),
$$

where $M_{0}=\rho \cdot 2 b^{0} \cdot U_{0}^{2}$; it is the momentum flux of the nozzle section.

In the impinging zone, the jet velocity $U_{m}$, the section length size $b_{u}$, and the pressure distribution $P_{m}$ exist as follows:

$$
\begin{gathered}
U_{m}, b_{u}=f_{3,4}\left(M_{0}, \rho, x, H\right), \\
P_{m}=f_{5}\left(M_{0}, \rho, x, H\right) .
\end{gathered}
$$

By using dimensional analysis and referring to relevant research conclusions in similar fields [34], we establish the following:

$$
\begin{gathered}
\frac{U_{m}}{U_{0}} \sqrt{\frac{H}{2 b_{0}}}=g_{1}\left(\frac{x}{H}\right) . \\
\frac{b_{u}}{H}=g_{2}\left(\frac{x}{H}\right) . \\
\frac{P_{m}}{\rho U_{0}^{2} / 2} \frac{H}{2 \mathrm{~b}_{0}}=g_{3}\left(\frac{x}{H}\right) .
\end{gathered}
$$

For the free jet zone, the following could be obtained:

$$
\frac{U_{m f}}{U_{0}} \sqrt{\frac{x}{2 b_{0}}}=C
$$

According to the simultaneous equations (14) and (17), the following results can be obtained:

$$
\frac{U_{m}}{U_{m f}}=h\left(\frac{x}{H}\right) .
$$

Based on the analysis of the pressure in the jet impinging zone, combined with equation (16), the pressure value at the 


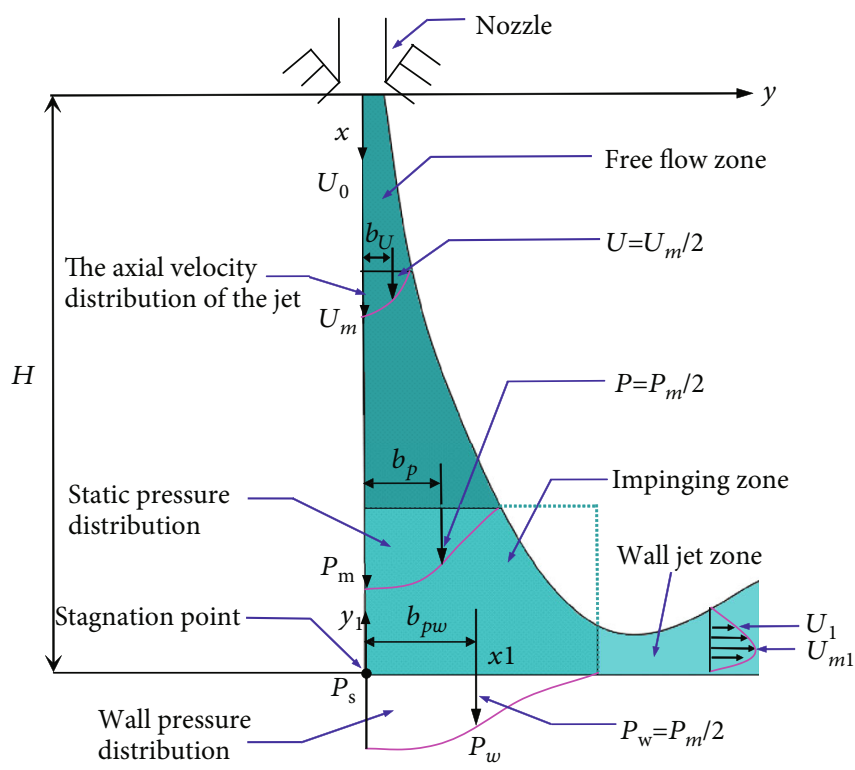

Figure 4: The coordinate system of gas jet development stage.

stagnation point can be obtained as follows:

$$
\frac{P_{s}}{\rho U_{0}^{2} / 2} \cdot \frac{H}{2 b_{0}}=g_{3}\left(\frac{x}{H}=1\right) .
$$

According to the simultaneous equations (16) and (19), the following results can be obtained:

$$
\frac{P_{m}}{P_{s}}=g_{4}\left(\frac{x}{H}\right)
$$

When the jet impinges on the coal, the wall pressure distribution at $x_{1}$ away from the jet axis is as follows:

$$
\frac{P_{w}}{\rho U_{0}^{2}} \frac{H}{2 b_{0}}=g_{5}\left(\frac{x_{1}}{H}\right) .
$$

For the simultaneous equations (19) and (21), the pressure on the inner wall of the impinging zone has the following relationship with the pressure at the stagnation point:

$$
\frac{P_{w}}{P_{s}}=g_{6}\left(\frac{x_{1}}{H}\right)
$$

According to the conclusion in Beltaos and Rajaratnam's work [35], equation (22) can be written as follows:

$$
\frac{P_{w}}{P_{s}}=e^{-38.5\left(x_{1} / H\right)^{2}} .
$$

The pressure distribution of the jet acting on the wall of the coal body is directly related to the feasibility of the jet breaking coal. The jet velocity and jet pressure in the impinging zone are analyzed as follows:

$$
\begin{gathered}
\frac{\partial}{\Delta y}(u v)_{y \rightarrow 0}=-K_{1}, \\
\left(u \frac{\Delta v}{\Delta y}+v \frac{\Delta u}{\Delta y}\right)_{y \longrightarrow 0}=-K_{1} .
\end{gathered}
$$

Equation (25) can be written by using the following continuity equation:

$$
\left(-u \frac{\Delta u}{\Delta x}+v \frac{\Delta u}{\Delta y}\right)_{y \longrightarrow 0}=-K_{1}
$$

When $y$ is 0 , in the direction of the jet axis, we have

$$
\frac{d}{d_{x}} U_{m}^{2}=2 K_{1}
$$

The integral treatment of equation (28) is as follows:

$$
U_{m}^{2}=2 K_{1} x+K_{2}
$$

Combining with equation (15), the axial velocity distribution of the jet flow can be written as follows:

$$
\begin{gathered}
\left(\frac{U_{m}}{U_{0}}\right)^{2} \frac{H}{2 b_{0}}=K_{3}\left(\frac{x}{H}\right)+K_{4}, \\
\left(\frac{U_{m}}{U_{0}}\right)^{2} \frac{H}{2 b_{0}}=K_{5}^{2}\left(1-\frac{x}{H}\right)+K_{6} .
\end{gathered}
$$

In combination with the law of momentum conservation, the pressure value at the stagnation point can be 


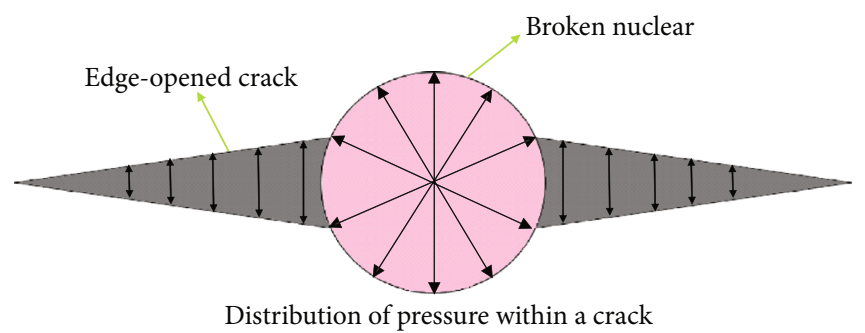

FIGURE 5: Schematic diagram of pressure inside a crack.

expressed as follows:

$$
P_{S}=\frac{\rho U_{0}^{2}}{2} \cdot \frac{2 b_{0}}{H} \cdot \frac{1}{K_{p w}},
$$

where $K_{p w}=\int_{0}^{\infty}\left(P_{w} / P_{s}\right) \cdot d\left(x_{1} / H\right)$, which can be set as 0.13 [34].

In the impinging zone, it is assumed that the turbulence phenomenon and gas viscosity effect caused by jet impinging can be ignored, and we have

$$
P_{w}+\frac{\rho U_{m 1}^{2}}{2}=P_{s}
$$

By combining equations (20) and (24), the velocity distribution along the $x_{1}$ direction of the wall surface can be obtained as follows:

$$
\frac{U_{m 1}}{U_{0}} \sqrt{\frac{H}{2 b_{0}}}=2.77\left[1-e^{-38.5\left(x_{1} / H\right) 2}\right]^{1 / 2} .
$$

2.2.3. Mathematical Model of Impact Stage. The ideal elasticplastic material failure process is different from the elasticplastic fracture theory [36] and forging extrusion theory [37]. This is because coal is a brittle material with dual medium characteristics of fracture and pore. When the instantaneous jet impinges on coal under a certain kinetic energy, the instantaneous jet velocity decreases rapidly and produces a back pressure gradient, which produces a blasting shock wave. Meanwhile, it strongly influences the energy compression force of coal, which destroys the coal wall and leads to the formation of a plastic compression crushing zone. Under the action of the blasting shock wave, stress waves are generated inside the coal and rock mass. Under the tensile action of the stress incident wave and the free surface reflected wave, the coal and rock mass are damaged to a certain extent. The particles inside the coal and rock mass are dislocated. Meanwhile, the medium units are tangentially stretched and radially compressed, leading to the further generation of an initial radial crack.

Given that the high-viscosity liquid water is different from the high-pressure gas jet in a continuous impact wave damage to the formation of a break zone and the initial crack, the low viscous gas flows quickly within the crack space. The pressure effect on the crack wall (Figure 5) follows the law of quasistatic pressure distribution, which can be approximately equal to the pressure of a jet under the action of the stagnation pressure [38]. The quasistatic pressure promotes the initial crack propagation, extension, and communication, and it generates a cross crack network inside the coal body. If the tensile stress of the gas in the fracture exceeds the tensile strength limit of the coal mass, the coal body will be destroyed and the coal particles will peel off, thus forming a plastic failure zone centered on the jet axis and forming erosion pits. The entry of the highpressure gas in these cracks decreases gradually after expansion, and the crack stops cracking when the pressure drops to the point where it is not sufficient to support further crack expansion.

Because the action time of the gas quasistatic pressure is much longer than that of the shock wave, it is generally believed that in the stage of gas jet impingement on rock breaking, quasistatic pressure failure is the main part, and tensile failure should be the main mechanism of gas jet impingement on coal pit formation.

For coal with jet action on the surface, the stress concentration in the crushing core and the extended crack is composed of the jet stagnation pressure and induced stress. The coal failure criterion can be defined by the following formula:

$$
P+\sigma_{t} \geq S_{t}
$$

where $P$ is the normal pressure acting on the crack. $P$ is the induced tensile stress generated and developed under the action of the jet impact. It can be understood as the stagnation pressure of the jet, and the induced pressure depends on the characteristics of the jet impact and the geometry of the crack.

Considering that an infinite plate is subjected to the vertical impact of a high-pressure gas jet (shown in Figure 5), the pressure distribution along the normal direction of the wall is $P=P(x, y)$. The stress distribution in the column coordinate system can be expressed as follows:

$$
\left\{\begin{array}{l}
\sigma_{r}=\frac{P}{2 \pi}\left\{(1-2 v)\left(\frac{1}{r^{2}}-\frac{Z}{r^{2}}\left(r^{2}+Z^{2}\right)^{-1 / 2}\right)-3 r^{2} Z\left(r^{2}+Z^{2}\right)^{-5 / 2}\right\}, \\
\sigma_{Z}=-\frac{3 P}{2 \pi} Z^{3}\left(r^{2}+Z^{2}\right)^{-5 / 2}, \\
\sigma_{\theta}=\frac{P}{2 \pi}(1-2 v)\left\{-\frac{1}{r^{2}}+\frac{Z}{r^{2}}\left(r^{2}+Z^{2}\right)^{-1 / 2}+Z\left(r^{2}+Z^{2}\right)^{-3 / 2}\right\},
\end{array}\right.
$$


In equation ((36)), $r$ is the radial distance from the $z$ -axis. The equation represents the pressure at any point of the object induced by the existence of normal pressure. When the pressure $P$ applied to the object is known, the stress induced by normal pressure can be obtained by integration. When a jet impinges on the flat panel wall surface, there is a point $Q$ on the surface of the panel. And $x$ and $y$ point to the tangent direction of point $Q$, respectively. The superposition principle is used at $Z=0$, as follows:

$$
\left\{\begin{array}{l}
\sigma_{x}=\left(\sigma_{r}(l, \theta) \cos \theta^{2}+\sigma_{\theta}(l, \theta) \sin \theta^{2}\right) l d l d \theta \\
\sigma_{y}=\left(\sigma_{r}(l, \theta) \sin \theta^{2}+\sigma_{\theta}(l, \theta) \cos \theta^{2}\right) l d l d \theta
\end{array}\right.
$$

The two formulas of equation (37) are divided into the local term with $Z$ and the nonlocal term without $Z$. The local term can be expressed as follows:

$$
\left\{\begin{array}{l}
\sigma_{r 1}=\frac{P}{2 \pi}\left\{(1-2 v)\left(-\frac{Z}{r^{2}}\left(r^{2}+z^{2}\right)^{-1 / 2}\right)-3 r^{2} Z\left(r^{2}+Z^{2}\right)^{-5 / 2}\right\}, \\
\sigma_{\theta 1}=\frac{P}{2 \pi}(1-2 v)\left\{\frac{Z}{r^{2}}\left(r^{2}+z^{2}\right)^{-1 / 2}+Z\left(r^{2}+Z^{2}\right)^{-3 / 2}\right\} .
\end{array}\right.
$$

The analysis of the above equation shows that the value of $Z$ is very small, and the values on the right side of the equation are very small except the value near point $Q$. Since the pressure distribution around point $Q$ is not obvious, it is approximately considered that the pressure is equal to that at point $Q$. In this case, the pressure is not affected. By integrating equation (37), we can obtain the following:

$$
\sigma_{x 1}=\sigma_{y 1}=\frac{P_{Q}}{2 \pi}\left[-(1-2 v)\left(l^{2}+Z^{2}\right)^{-5 / 2} \times 3 \pi r^{2} Z+Z\left(l^{2}+Z^{2}\right)^{-3 / 2} \pi\right] l d l .
$$

By integrating the above formula within a circle with a small radius (when $l$ is set to $a$ ), we can get the following:

$$
\sigma_{x 1}=\sigma_{y 1}=\frac{P_{Q}}{2}\left[-(1+2 v)+\frac{2(1+2 v) Z}{\sqrt{a^{2}+Z^{2}}}-\left(\frac{Z}{\sqrt{a^{2}+Z^{2}}}\right)^{3}\right] .
$$

When $Z$ approaches 0 , there is

$$
\sigma_{x 1}=\sigma_{y 1}=-\frac{P_{Q}}{2}(1+2 v) .
$$

For the nonlocal phase, there is

$$
\sigma_{x 2}=-\sigma_{y 2}=\frac{1}{2 \pi}(1-2 v)-\frac{P(x, y)}{l^{2}}\left(\cos \theta^{2}-\sin \theta^{2}\right) l d l d \theta .
$$

Considering that the value of the nonlocal phase is much smaller than that of the local phase and the value of the nonlocal phase gradually disappears as it approaches the axis direction, only local terms are generally considered. Combining the local stress failure criterion and local terms, the relationship between jet pressure and Poisson's ratio and tensile strength of a coal body under the tensile failure criterion of a coal body would be established as follows:

$$
P \geq \frac{2}{1-2 \nu} S_{t}
$$

2.2.4. Criteria for Identification of Coal Breaking Capacity and Erosion Pit Characteristics by Gas Jet. Combining with the mathematical model that illustrates the continuous process of coal breaking by a gas jet, when the jet pressure is greater than the critical failure pressure of coal, erosion holes will be formed. After combining equations (9), (11), (32), and (43), the criteria for determining whether a gas jet can break coal can be obtained:

$$
\begin{gathered}
\frac{7.7 k R b_{0}}{H} \cdot \frac{T}{\left(P^{*} / P\right)^{(k-1 / k)}} \cdot \frac{\rho}{\left(P^{*} / P\right)^{1 / k}} \\
\cdot \frac{2}{k-1}\left[\left(\frac{P^{*}}{P}\right)^{(k-1 / k)}-1\right] \geq \frac{2}{1-2 v} S_{t},
\end{gathered}
$$

where the adiabatic coefficient $k$ is 1.4 for air, the gas constant $R$ is $287.06 \mathrm{~J} /(\mathrm{kg} \cdot \mathrm{k})$ for air, $b_{0}$ is the section area of the nozzle outlet, $H$ is the initial jet impact distance, and other relevant parameters are defined as equation (9). The ratio of gas stagnation parameters to static parameters is defined as follows:

$$
a=\left(\frac{P^{*}}{P}\right)^{1 / k}
$$

After the simplification of equation (44), we can obtain the following:

$$
\frac{1.54 \times 10^{4} T \rho b_{0}\left(a^{0.4}-1\right)}{H \cdot a^{1.4}} \geq \frac{2}{1-2 v} S_{t} .
$$

When the coal particles are eroded and stripped by a jet, the impact distance of the jet gradually increases, and the pressure value at the stagnation point gradually decreases. When the pressure is less than the critical failure pressure of coal and rock mass, the jet is insufficient to cause damage to the coal body. Under the premise that the nozzle geometric parameters, gas state parameters, and coal mechanics parameters are known, the initial impact distance of the jet is determined, and the axial depth of the hole formed by jet erosion can be obtained from the following formula:

$$
\begin{aligned}
& \Delta h=\frac{k R b_{0}(1-2 v)}{(k-1) S_{t} k_{p w}}\left[\left(\frac{P^{*}}{P}\right)^{(k-1 / k)}-1\right] \frac{T \rho P}{P^{*}}-H, \\
& \Delta h=\frac{7.7 \times 10^{3} T \rho b_{0}(1-2 v) \cdot\left[a^{0.4}-1\right]}{S_{t} a^{1.4}}-H .
\end{aligned}
$$


TABLE 1: The theoretical values of the parameters under different jet pressures.

\begin{tabular}{lcccccccc}
\hline$P_{2}(\mathrm{MPa})$ & $T(\mathrm{~K})$ & $\mathrm{Ma}$ & $H(\mathrm{~mm})$ & $P_{w}(\mathrm{MPa})$ & $P_{s}(\mathrm{MPa})$ & $U_{0}(\mathrm{~m} / \mathrm{s})$ & $\Delta h(\mathrm{~mm})$ & $x(\mathrm{~mm})$ \\
\hline 2 & 298 & 3.5 & 30 & 2.667 & 54.181 & 652.104 & 3.647 & 1.80 \\
4 & 298 & 3.5 & 30 & 2.667 & 108.362 & 652.104 & 37.293 \\
6 & 298 & 3.5 & 30 & 2.667 & 162.543 & 652.104 & 70.940 & 4.99 \\
8 & 298 & 3.5 & 30 & 2.667 & 216.724 & 652.104 & 94.587 & 4.69 \\
10 & 298 & 3.5 & 30 & 2.667 & 270.905 & 652.104 & 108.234 \\
12 & 298 & 3.5 & 30 & 2.667 & 325.086 & 652.104 & 111.880 & 5.13 \\
\hline
\end{tabular}

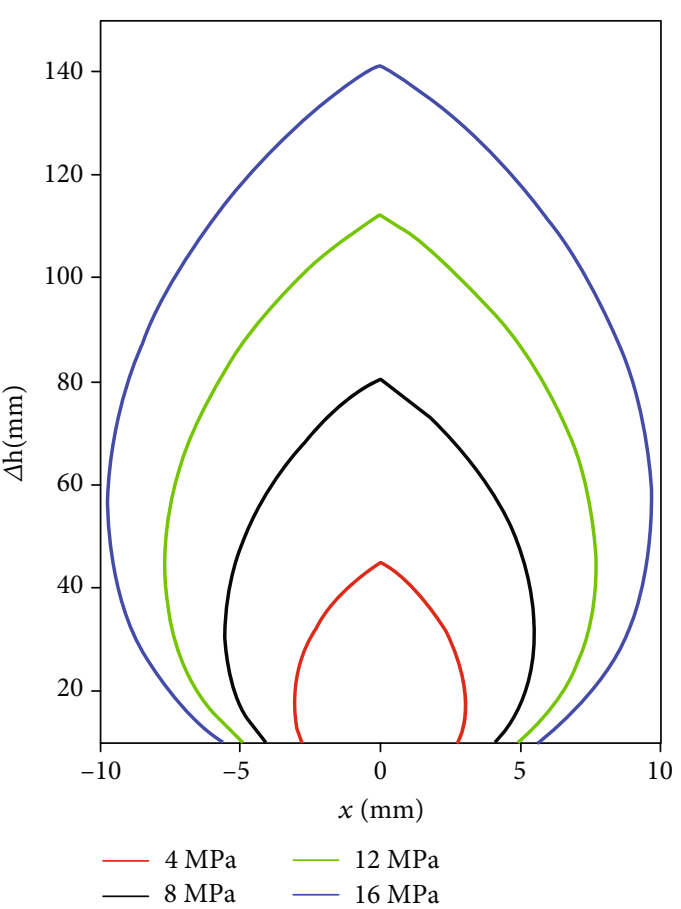

Figure 6: Characteristics of erosion pit under different jet conditions.

Table 2: Pressure at pipeline inlet under different jet pressures.

\begin{tabular}{lcccccc}
\hline$P_{2}(\mathrm{MPa})$ & $l(\mathrm{~m})$ & $d(\mathrm{~m})$ & $A\left(\mathrm{~m}^{2}\right)$ & $\lambda$ & $G$ & $P_{1}(\mathrm{~Pa})$ \\
\hline 2 & 1000 & 0.2 & 0.0314 & 1.6 & 0.47 & 2000037.43 \\
4 & 1000 & 0.2 & 0.0314 & 1.6 & 0.94 & 4000074.87 \\
6 & 1000 & 0.2 & 0.0314 & 1.6 & 1.40 & 6000112.30 \\
8 & 1000 & 0.2 & 0.0314 & 1.6 & 1.87 & 8000149.73 \\
10 & 1000 & 0.2 & 0.0314 & 1.6 & 2.34 & 10000187.17 \\
12 & 1000 & 0.2 & 0.0314 & 1.6 & 2.81 & 12000224.60
\end{tabular}

In terms of the radial pressure distribution of the jet impact on the coal, when the pressure is greater than the critical failure pressure, the radius of the erosion hole at the corresponding impact distance can be calculated as follows:

$$
x=\frac{7.7 \times 10^{3} T \rho b_{0}\left[a^{0.4}-1\right]}{S_{t} \cdot(1-2 v)^{-1} a^{1.4}} \cdot \sqrt{\frac{\log \left(P_{w}^{*} / P_{s}^{*}\right)}{-38.5}}
$$

where $P_{w}^{*}$ represents the critical failure pressure of the
TABle 3: Mass flow rate at nozzle outlet under different jet pressures.

\begin{tabular}{lccc}
\hline$P_{2}(\mathrm{MPa})$ & $T(\mathrm{~K})$ & Radius of nozzle throat, $b_{1}(\mathrm{~mm})$ & $m(\mathrm{~kg} / \mathrm{s})$ \\
\hline 2 & 298 & 1 & 0.0147 \\
4 & 298 & 1 & 0.0294 \\
6 & 298 & 1 & 0.0441 \\
8 & 298 & 1 & 0.0588 \\
10 & 298 & 1 & 0.0735 \\
12 & 298 & 1 & 0.0882 \\
\hline
\end{tabular}

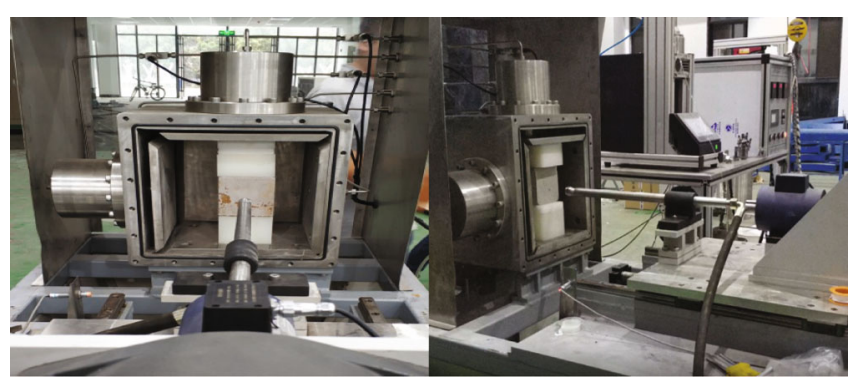

Figure 7: Physical diagram of a test block fixing device.

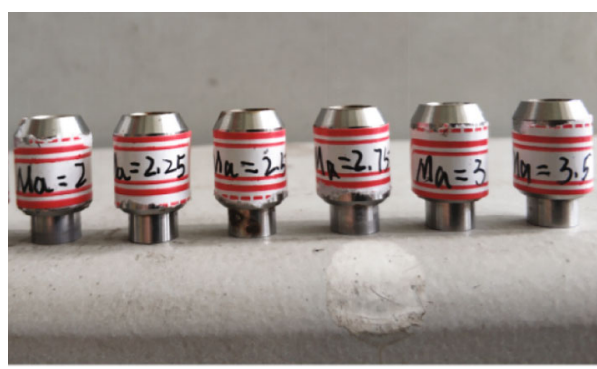

Figure 8: Physical diagram of nozzle.

wall under the action of the jet impact, and $P_{s}^{*}$ represents the pressure value at the stagnation point under the impact distance $H$.

It can be seen from equations (48) and (49) that, in addition to environmental factors, the coal-fracturing capacity of a gas jet could be also affected by jet pressure, target distance, nozzle radius, and coal strength.

2.3. Mass Flow Rate at Nozzle Outlet. According to the continuity equation of gas steady flow, the flow rate of gas is the same through any section of the nozzle. However, the flow rate of all kinds of nozzles is limited by their minimum 
TABLE 4: Material ratio and mechanical parameters.

\begin{tabular}{lcccccrrr}
\hline No. & Cement & Gypsum & Sand & Water & Sand cement ratio & Water solid ratio & $S_{t}$ & $v$ \\
\hline 1 & 3 & 1 & 10 & 3 & 2.5 & 0.214 & 0.64 & 0.24 \\
2 & 3 & 1 & 8 & 2 & 2.0 & 0.167 & 0.70 \\
3 & 3 & 1 & 6 & 1.5 & 1.5 & 0.26 \\
4 & 1 & 3 & 8 & 2 & 2.0 & 0.150 & 0.78 \\
\hline
\end{tabular}

cross-section. Therefore, the flow rate is usually calculated according to the minimum cross-section $[39,40]$.

$$
q_{m}=S a^{*} \rho^{*}
$$

where $S$ is the area of the nozzle throat; $a^{*}$ is the velocity of the jet in the throat, and $\rho^{*}$ is the density of the jet in the throat.

When the gas velocity through the nozzle reaches the speed of sound, the gas mass flow will reach the maximum value. In this work, the gas jet reaches the speed of sound at the nozzle throat. Therefore, the mass flow at the nozzle outlet is equal to that speed at the throat.

\section{Theoretical Calculation and Experimental Verification}

3.1. Theoretical Calculation. In order to preliminarily analyze the coal-breaking capacity of a gas jet and the law of pit formation, the coal-breaking law of a gas jet is theoretically calculated according to an established mathematical model. Due to space limitations, only the theoretical values of the parameters under different jet pressures are listed in Table 1.

In order to visually investigate the characteristics of erosion pits formed by a gas jet impinged on coal and rock mass, the initial impact distance is selected to be $30 \mathrm{~mm}$, and the nozzle specification is $3 \mathrm{Ma}$. According to equations (48) and (49), the shape of erosion pits under different jet pressures is calculated, and the results are shown in Figure 6. With the increase of the pressure at the nozzle inlet, the depth and radial length of erosion pits formed by a jet impact increase continuously, but the increase of the axial depth of holes is much larger than that of radial length. When the pressure at the nozzle inlet increases, the jet impact kinetic energy increases and the gas stagnation pressure acting on the crack increases, which is reflected in the expansion of the axial and radial crushing range of the erosion hole. Because the wall pressure attenuates rapidly from the axial direction, the increment of hole radial length is much smaller than that of axial depth. Further observation shows that with the increase of nozzle inlet pressure, the shape of the erosion hole formed by the jet impact on the same coal and rock mass gradually changes from the initial conical shape to an elliptical shape.

For the pipeline part, a new seamless steel tube is selected as the research object (at this point, the absolute friction degree of the pipeline $\varepsilon=0.15$ ). Under the condition that the temperature $T=298 \mathrm{~K}$ and the outlet pressure of the pipeline is known as the inlet pressure of the nozzle, the pressure value at the inlet of the pipeline is calculated, as shown in Table 2.

It can be found from Table 2 that the pressure at the outlet of the pipeline is slightly higher than that at the inlet under the assumption that the gas flows in the pipeline are in a constant temperature environment.

In the case of only changing the jet pressure, the mass flow rate at the nozzle outlet under different jet parameters is calculated, as shown in Table 3.

It is found from the Table 3 that the mass flow rate at the nozzle outlet only increases with the increase of jet pressure under the condition of constant external back pressure.

3.2. Experimental Verification of the Size of the Erosion Pit. According to the established mathematical model, the theoretical values of the depth and radius of the erosion pit under different jet parameters are calculated and verified by experiments. Considering the difficulty and safety of obtaining gas raw materials, air was selected for the experiment in this task. The following analyses include the influence of Mach number $\mathrm{Ma}$, target distance $H$, jet pressure $P$, and tensile strength $S_{t}$ on the coal-breaking ability of a gas jet.

3.2.1. Experimental System. As shown in Figure 1, the experimental system consists of three parts: high-pressure supersonic gas jet forming and controlling device, test block fixing device, and nozzle system. These three parts of the experimental system are independent of each other in structure and are easy to assemble and disassemble. They are connected with each other through a high-pressure rubber hose and a high-pressure steel pipe. When the gas is repeatedly compressed by the air compressor, the normal temperature and atmospheric pressure gas is stored in the high-pressure gas storage tank. After the manual pressure regulating valve is opened, the high-pressure gas flows through the pipeline and nozzle and ejects outwards. Then, the jet continuously impacts on the wall of the coal and rock mass, resulting in erosion damage. The following are the details of the experiment.

(1) The High-Pressure Supersonic Gas Jet Forming and Controlling Device. The gas jet forming and controlling device in the system is composed of a piston air compressor, a highpressure gas storage tank, a high-pressure pipeline, a pressure gauge, and a pressure regulating valve. The air with normal temperature and atmospheric pressure is first repeatedly pressurized by the air compressor and then stored in the high-pressure gas storage tank. The pressure is read by the pressure gauge installed on the intake pipeline, and the 


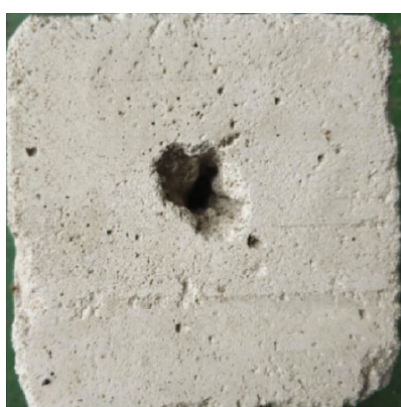

(a) $H=30 \mathrm{~mm}$

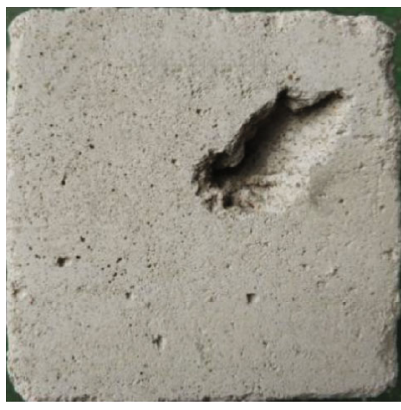

(c) $H=50 \mathrm{~mm}$

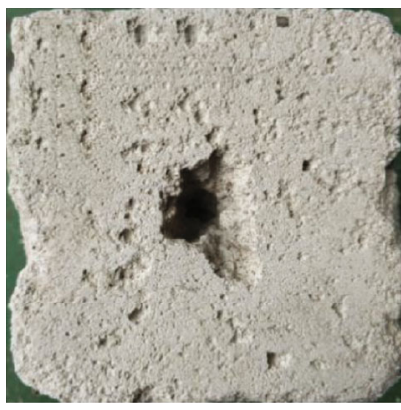

(e) $\mathrm{Ma}=2.75$

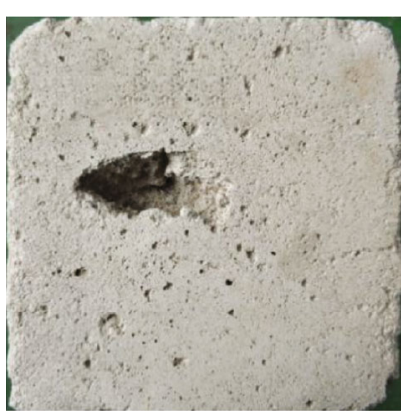

(g) $P=4 \mathrm{MPa}$

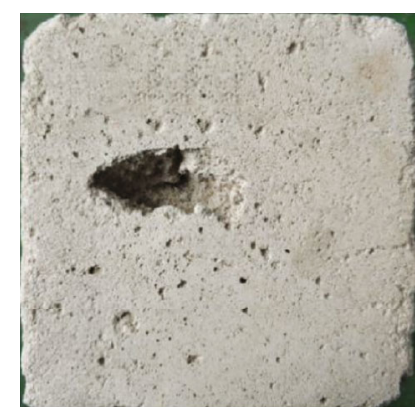

(b) $H=40 \mathrm{~mm}$

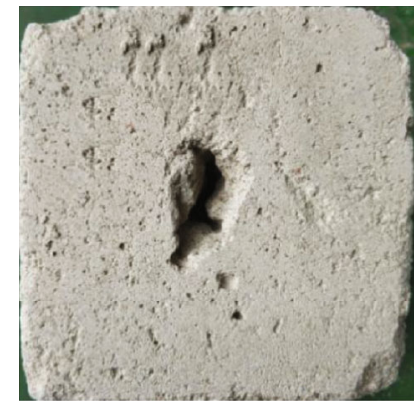

(d) $\mathrm{Ma}=2$

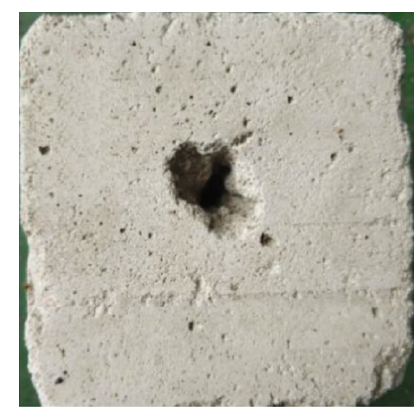

(f) $\mathrm{Ma}=3.5$

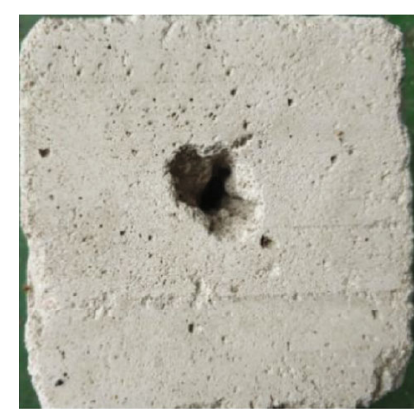

(h) $P=8 \mathrm{MPa}$

Figure 9: Continued. 


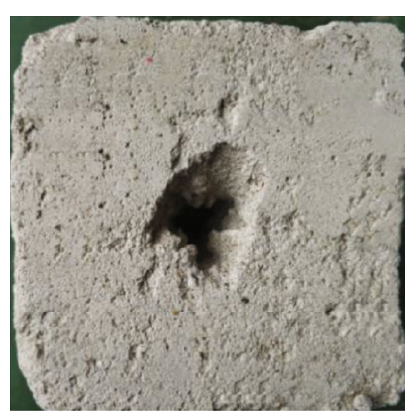

(i) $P=12 \mathrm{MPa}$

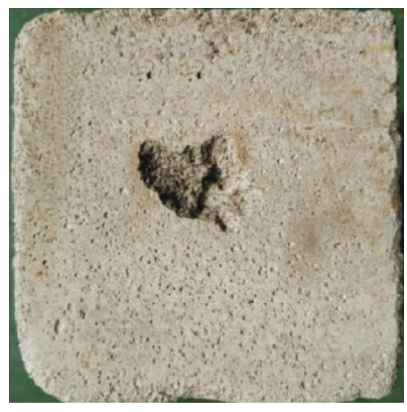

(k) $S_{t}=0.4 \mathrm{MPa}$

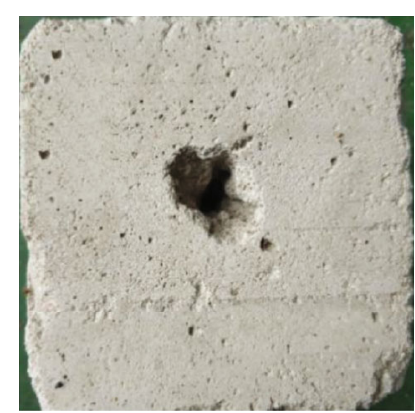

(j) $S_{t}=0.3 \mathrm{MPa}$

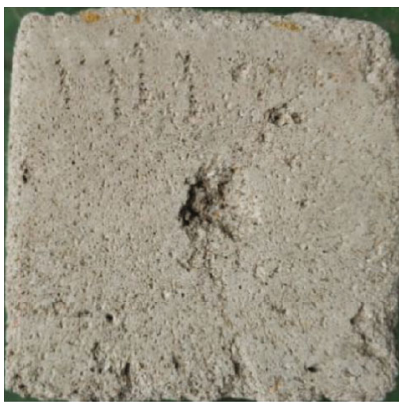

(l) $S_{t}=0.5 \mathrm{MPa}$

Figure 9: Shape of erosion pit formed by different jet parameters (Ma, $H, P, S_{t}$ ).

start-up and stop of the air compressor are controlled. When the specified pressure is reached, the required gas pressure is manually adjusted by the pressure regulating valve.

(2) Test Block Fixing Device. Due to the large impact dynamic pressure in the process of gas jet impingement, it is necessary to fix the test block in order to ensure that the test block is not displaced in the impact process. The RLCJ-1 device produced by Nantong Renlong Scientific Research Instrument Co., Ltd. was used to fix the test block. The loading pressure is $0-60 \mathrm{MPa}$, the loading accuracy is $0.01 \mathrm{MPa}$, and the size of loading chamber is $250 \mathrm{~mm} \times 250$ $\mathrm{mm} \times 300 \mathrm{~mm}$. Because the size of the test block made of similar material is $100 \mathrm{~mm} \times 100 \mathrm{~mm} \times 100 \mathrm{~mm}$ and the stroke of the piston column of the loading device is limited $(30 \mathrm{~mm})$, in order to load the pressure to similar materials, adding a gasket in the confining pressure loading chamber was considered. Because the cabin space is larger than that of similar materials, the size of the high-strength finish rolling pad commonly used in confining pressure loading should also be large enough. Considering the convenience of the experimental process, a high molecular polyethylene backing plate is used. The physical figure is shown in Figure 7.

(3) Nozzle System. In order to realize supersonic flow, the jet velocity must be realized by the Laval nozzle. In this test, a high-pressure rubber pipe and a high-pressure steel pipe were installed at the outlet of the pressure regulating valve, and the screw thread was processed at the end of the highpressure steel pipe to connect the Laval nozzle. The nozzle specifications include $2 \mathrm{Ma}, 2.25 \mathrm{Ma}, 2.5 \mathrm{Ma}, 2.75 \mathrm{Ma}, 3 \mathrm{Ma}$,
3.25 Ma, and 3.5 Ma. The inlet diameter of the nozzle is $7 \mathrm{~mm}$, and the rear diameter is $2 \mathrm{~mm}$. The outlet diameter is different. The larger the Mach number, the larger the section diameter of the corresponding nozzle outlet. The physical figure is shown in Figure 8.

\subsubsection{Experimental Method and Sample Preparation. The} pressure regulating valve is adjusted to the required experimental pressure, the impact target distance and the nozzle specification of the nozzle are adjusted, the air outlet valve is opened, and the high-pressure gas flows through the nozzle to form a high-pressure supersonic gas jet, causing erosion damage to the sample. After the jet pressure is significantly reduced, the valve is closed. After impact, a vernier caliper is used to measure the depth and width of the erosion pit. After the measurement, the first test is completed. Other different test blocks are replaced for repeated tests.

In order to achieve good results in the jet impingement experiment, raw coal is the best impact material. However, due to the randomness of the development of the actual coal joints and fissures, the mechanical structure is complex, and it is very easily broken in the process of sampling and sample preparation. It is difficult to obtain coal blocks with regular or similar mechanical parameters, and the number of test blocks required in the experiment is large. Therefore, cement, gypsum, river sand, and other similar materials are considered as a replacement for raw coal for processing. According to the mechanical parameters of raw coal, the proportion of raw materials is carried out to ensure that the stress-strain, compressive strength, tensile strength, elastic modulus, and Poisson's ratio of similar materials are as 


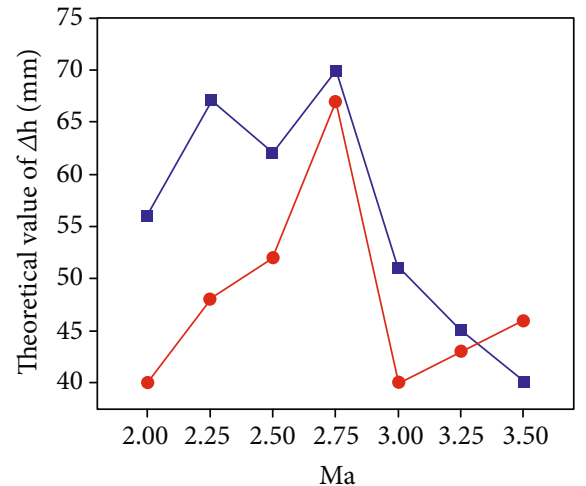

(a)

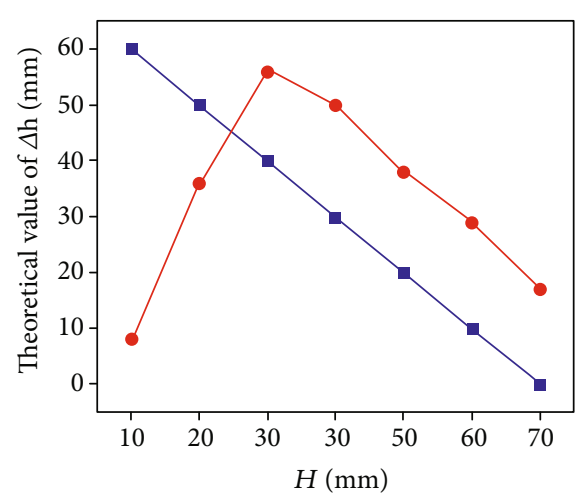

(c)

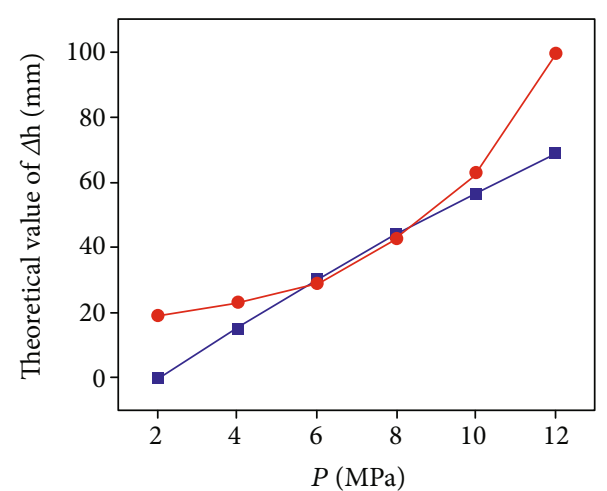

(e)

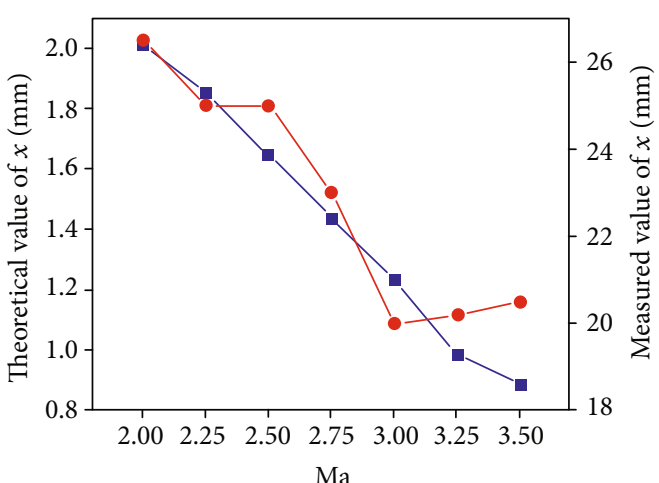

(b)

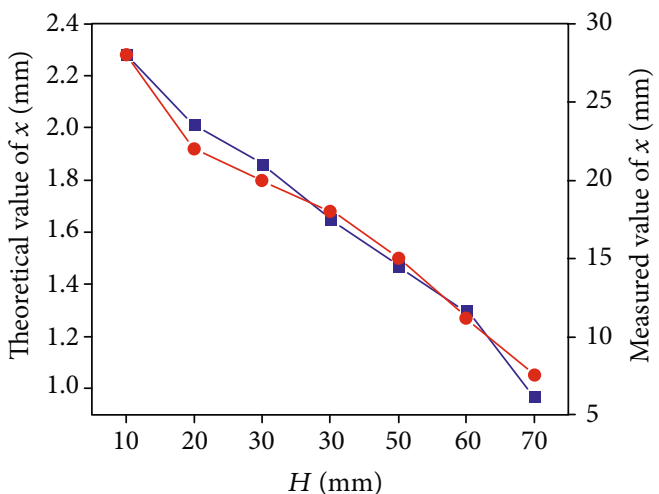

(d)

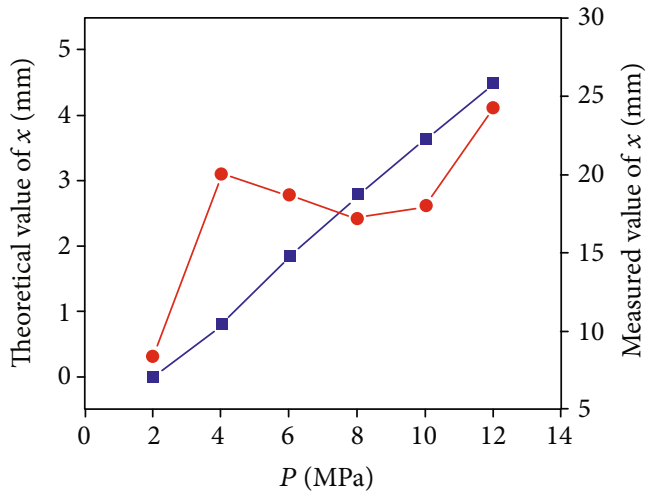

(f)

FIgURE 10: Continued. 


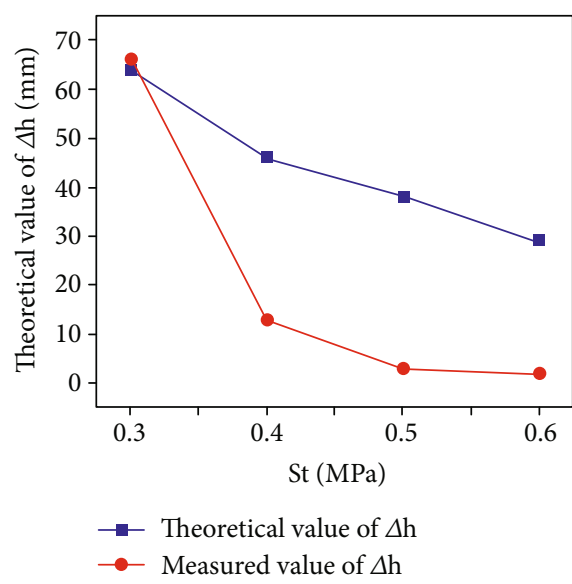

(g)

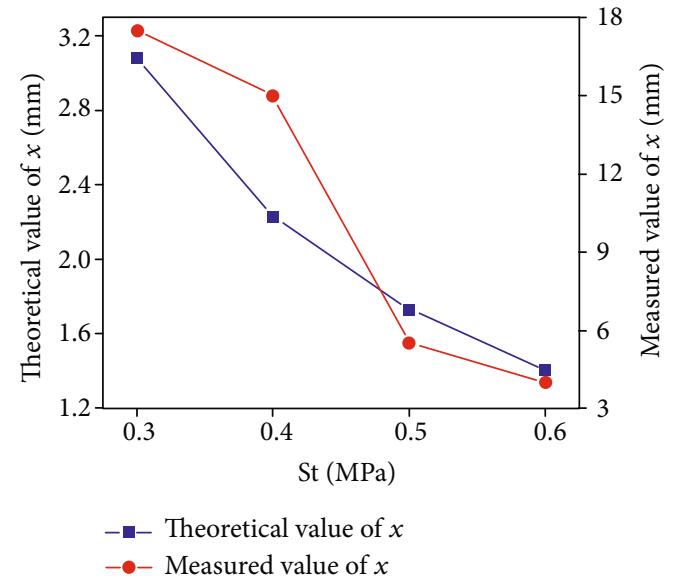

(h)

Figure 10: Theoretical and measured values of erosion pit size under different fracture parameters (Ma, H, P, St).

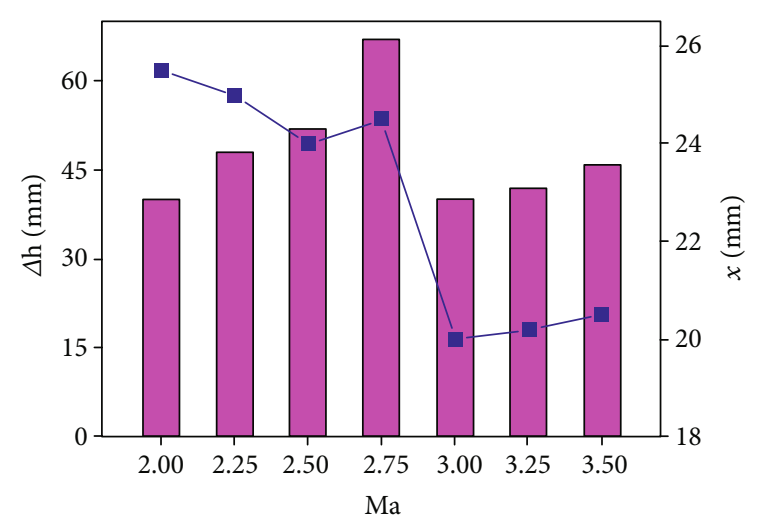

(a)

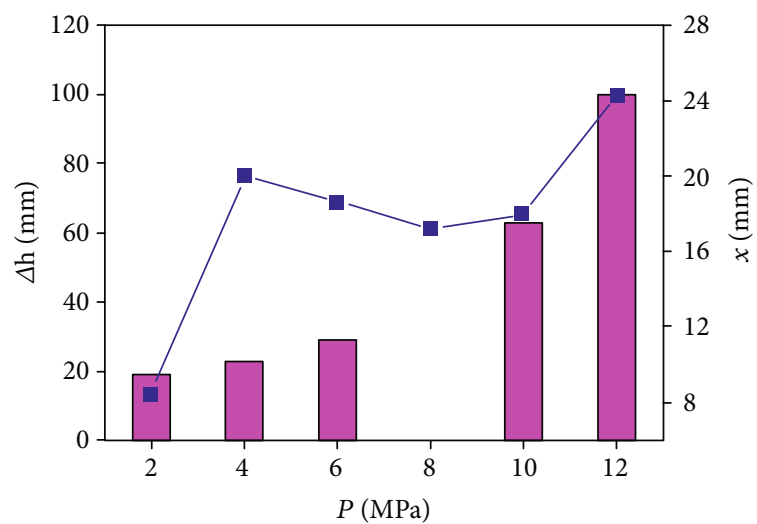

$\underset{-\square-x}{\square} \Delta \mathrm{h}$

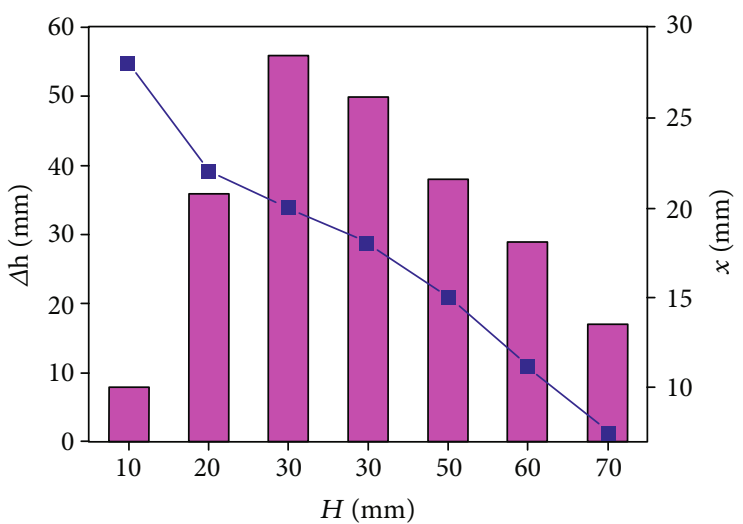

(b)

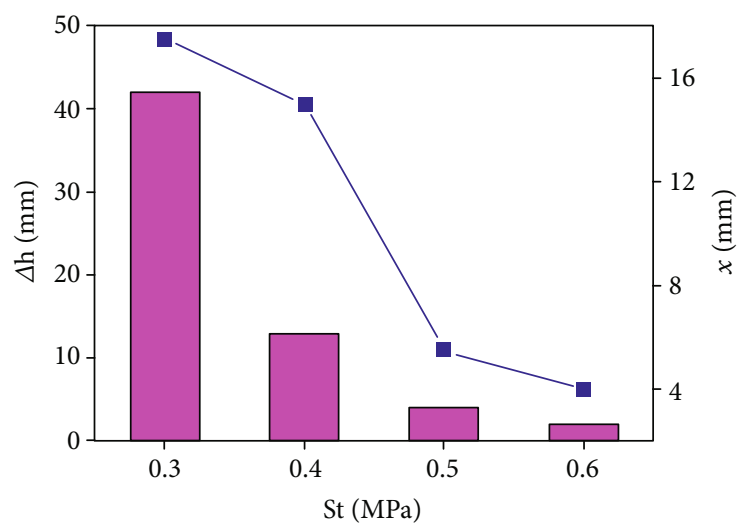

(d)

Figure 11: Variation of depth and radius of an erosion pit with different jet parameters $\left(\mathrm{Ma}, H, P, S_{t}\right)$.

close as possible to the raw coal, as shown in Table 4 . The size of the test block is $100 \mathrm{~mm} \times 100 \mathrm{~mm} \times 100 \mathrm{~mm}$ for experimental verification.
3.2.3. Verification. After the installation of the experimental device, we carried out four groups of experiments under the condition of changing the jet parameters. The following 
analyses include the influence of Mach number $\mathrm{Ma}(2,2.25$, $2.5,2.75,3,3.25,3.5 \mathrm{Ma})$, target distance $H(10,20,30,40$, $50,60$, and $70 \mathrm{~mm})$, jet pressure $P(2,4,6,8,10$, and $12 \mathrm{MPa})$, and tensile strength $S_{t}(0.3,0.4,0.5$, and $0.6 \mathrm{MPa})$ on the coal-breaking ability of a gas jet. The size of the erosion pit directly reflects the coal-breaking ability of each jet parameter, so the radius and depth of the erosion pit are taken as the comparison parameters. The sample after the experiment is shown in Figure 9. After measuring the erosion pit, the experimental value is compared with the theoretical value, and the results are shown in Figure 10.

As shown in Figure 10, the theoretical and measured values under some parameters have roughly the same variation trend after ignoring the large error in the experimental data. However, with the increase of Mach number and target distance, the theoretical value of erosion pit depth decreases gradually, while the measured value increases first and then decreases. With the increase of jet pressure, the change in the radius of an erosion pit is opposite to the Mach number and target distance.

Differently from the monotonic variation of theoretical values, there is a turning point in the variation of experimental values under some parameters. Taking the variation under different target distances as an example, the depth of the erosion pit increases with the increase of the target distance and reaches the deepest depth when the target distance is $30 \mathrm{~mm}$. The radius of the erosion pit also remains almost unchanged after the target distance is $30 \mathrm{~mm}$. Therefore, the optimal impinging distance is $30 \mathrm{~mm}$, rather than that the smaller distance would cause a better impinging effect.

In order to determine these optimal values within the paper's research scope, experimental data under various jet parameters are analyzed, and the results are shown in Figure 11.

Under the condition of a constant nozzle Mach number and impingement distance, the depth of an erosion pit formed by high-pressure gas jet impact and the area of an erosion pit both increases with the increase of nozzle inlet pressure. Considering the influence of the depth and radius of an erosion pit comprehensively, certain rock breaking can be achieved under the action of jet impingement. The impact pressure of a gas jet should be increased continuously.

Under the condition of a constant nozzle inlet pressure and nozzle Mach number, the depth of an erosion pit formed by high-pressure gas jet impact first increases and then decreases with the increase of target distance. Besides the large area peeling data of test block surface with $10 \mathrm{~mm}$ spacing, the erosion pit area also shows the trend of first increasing and then decreasing. It is considered that an optimum distance of $30 \mathrm{~mm}$ exists under the action of jet impingement.

Under the condition of a constant nozzle inlet pressure and impingement distance, the depth of an erosion pit formed by high-pressure jet impingement increases with the increase of nozzle Mach number, and the erosion pit area reaches the maximum when the nozzle Mach number is $2.75 \mathrm{Ma}$. Considering the influence of the depth and radius of the erosion pit, the rock-breaking effect is better when the nozzle size is $2.75 \mathrm{Ma}$.
Under the condition of constant nozzle inlet pressure, nozzle Mach number, and impingement distance, the depth of an erosion pit formed by high-pressure gas jet impact and the area of an erosion pit both decreases with the increase of coal strength.

\section{Discussion}

Gas jet breaking coal and rock is a new type of pressure relief and permeability increasing technology. By studying the continuous action mechanism of a gas jet and the influence law of different jet parameters on erosion and coal breaking, the theory of gas jet impacting coal and rock breaking is enriched and developed, and the selection of jet parameters is optimized, which is of great significance for the application of gas jet engineering. Although the mechanism and law of coal breaking by gas jet impingement have been studied in this work, there are still some deficiencies which need to be further studied and improved. (1) Limited by the influence of a high-pressure gas source, it is impossible to realize a high-pressure and long-time jet impingement experiment. In the selection of experimental factors, the influence of impact time on the experimental results is ignored, and the optimal value of jet parameters cannot be completely accurately selected. (2) Because the raw coal sample is not easily obtained and its demand is usually large, it is replaced by similar materials in this work. The results show that the similar materials do not have the joint structure of raw coal, and the experimental results will have a certain degree of deviation. Moreover, raw coal exists in the complex stress field, which is not easily achieved in the laboratory conditions. Therefore, the field test of a gas jet breaking coal and rock is needed in our future research.

\section{Conclusion}

(1) The mathematical model of the full impinging process of coal and rock fracturing by a supersonic gas jet is established. In particular, the transport of high-pressure gas in a pipeline is included in the model. The relationship between the pressure at the inlet and the pressure at the outlet of the pipeline is obtained, and the remote supply of high-pressure gas can be achieved. Moreover, the criteria for distinguishing the coal-fracturing ability of a gas jet and the characteristics of an erosion pit are deduced, and it is found that the shape of the erosion pit is ellipse, which lays a foundation for further theoretical research and field process design

(2) Laboratory experiment results show that different from the monotonic variation of theoretical values, a turning point exists in the variation of experimental values under some parameters. Taking the variation under different target distances as an example, the depth of the erosion pit increases with the increase of the target distance and reaches the deepest depth when the target distance is $30 \mathrm{~mm}$. The 
radius of the erosion pit also remains almost unchanged after the target distance is $30 \mathrm{~mm}$

(3) Considering the influence of the depth and radius of erosion pit comprehensively, certain rock-breaking effects can be achieved under the action of jet impingement. The impact pressure of a gas jet should be increased continuously. The rock-breaking effect is better when the nozzle size is $2.75 \mathrm{Ma}$

\section{Nomenclature}

g: $\quad$ Gravitational acceleration $\left(\mathrm{m} / \mathrm{s}^{2}\right)$

$u_{1}$ : $\quad$ Speed at pipeline inlet $(\mathrm{m} / \mathrm{s})$

$u_{2}$ : Speed at pipeline outlet $(\mathrm{m} / \mathrm{s})$

$p_{1}$ : $\quad$ Pressure at pipeline inlet $(\mathrm{Pa})$

$p_{2}$ : $\quad$ Pressure at pipeline outlet $(\mathrm{Pa})$

$h_{f}: \quad$ Resistance loss $(\mathrm{J} / \mathrm{kg})$

$\rho: \quad$ Density $\left(\mathrm{kg} / \mathrm{m}^{3}\right)$

$v$ : $\quad$ Specific volume of gas $\left(\mathrm{m}^{3} / \mathrm{kg}\right)$

$L: \quad$ Length of pipeline $(\mathrm{m})$

$d$ : $\quad$ Diameter of pipeline $(\mathrm{m})$

Re: $\quad$ Reynolds number

$\lambda: \quad$ Friction coefficient

$\mu: \quad$ Viscosity (Pa.s)

$q_{m}: \quad$ Mass flow rate $(\mathrm{kg} / \mathrm{s})$

A: $\quad$ Section area of pipeline $\left(\mathrm{m}^{2}\right)$

G: $\quad$ Mass flow rate $(\mathrm{kg} / \mathrm{s})$

R: $\quad$ Gas constant, $287.06 \mathrm{~J} /(\mathrm{kg} \cdot \mathrm{K})$ for air

T: $\quad$ Thermodynamic temperature $(\mathrm{K})$

$M: \quad$ Molar mass $(\mathrm{g} / \mathrm{mol})$

$S: \quad$ Any cross-sectional area of nozzle $\left(\mathrm{m}^{2}\right)$

Se: $\quad$ Nozzle throat area $\left(\mathrm{m}^{2}\right)$

Ma: Mach number

$\mathrm{Ma}_{\max }$ : Maximum Mach number

$k$ : $\quad$ Adiabatic coefficient, 1.4 for air

$P: \quad$ Gas pressure $(\mathrm{Pa})$

$P^{*}: \quad$ Gas pressure under stagnation state $(\mathrm{Pa})$

$\rho^{*}: \quad$ Gas density at stagnation state $\left(\mathrm{kg} / \mathrm{m}^{3}\right)$

$T^{*}$ : Thermodynamic temperature of gas in stagnation state $(\mathrm{K})$

a: $\quad$ Sound velocity $(\mathrm{m} / \mathrm{s})$

$b_{0}: \quad$ Radius at nozzle outlet $(\mathrm{m})$

$H$ : $\quad$ Target distance: the distance from the nozzle outlet to the plate $(\mathrm{m})$

$\theta: \quad$ Jet angle $\left({ }^{\circ}\right)$

$U_{0}$ : Initial velocity of a jet $(\mathrm{m} / \mathrm{s})$

$P_{m}: \quad$ Jet axial pressure $(\mathrm{Pa})$

$P_{s}: \quad$ Pressure at stagnation point $(\mathrm{Pa})$

$P_{s}^{*}: \quad$ Pressure value at stagnation point at impact distance $H(\mathrm{~Pa})$

$P_{w}: \quad$ Pressure at wall $(\mathrm{Pa})$

$P_{w}^{*}: \quad$ Critical failure pressure at the wall $(\mathrm{Pa})$

$U_{m}: \quad$ Jet velocity $(\mathrm{m} / \mathrm{s})$

$v$ : Poisson's ratio

$S_{t}: \quad$ Tensile strength $(\mathrm{Pa})$

$K_{p w}: \quad$ Constant (0.13)

$U_{m 1}$ : Maximum value of $U_{m}$ in $x_{1}$ direction of any crosssection $(\mathrm{m} / \mathrm{s})$ $b_{u}$ : Radial length dimension of fluid cross-section velocity of $1 / 2 U_{m}$ in a free jet section $(\mathrm{m})$

$U_{m f}: \quad$ Axial velocity of jet $(\mathrm{m} / \mathrm{s})$

$b_{f}: \quad$ Radial length of jet $(\mathrm{m})$

$x: \quad$ Radius of erosion pit $(\mathrm{m})$

$\Delta h$ : Depth of erosion pit (m).

\section{Data Availability}

The datasets used or analyzed during the current study are available from the corresponding author on reasonable request.

\section{Conflicts of Interest}

The authors declare that they have no known competing financial interests or personal relationships that could have appeared to influence the work reported in this paper.

\section{Authors' Contributions}

Gongda Wang performed formal analysis and wrote the original draft of the manuscript. Yuanyuan Wang conducted the investigation and wrote, reviewed, and edited the manuscript. Xin Yang performed formal analysis. Xin Song contributed in the conduct of the investigation.

\section{Acknowledgments}

This work was supported by National Natural Science Foundation of China (51974161, 51804161, 52074156, and 52004291) and the Chinese Postdoctoral Science Foundation (2019M660861).

\section{References}

[1] T. Moore, "Coalbed methane: a review," International Journal of Coal Geology, vol. 101, pp. 36-81, 2012.

[2] J. Liu, Z. Chen, D. Elsworth, H. Qu, and D. Chen, "Interactions of multiple processes during CBM extraction: a critical review," International Journal of Coal Geology, vol. 87, no. 34, pp. 175-189, 2011.

[3] C. Fan, D. Elsworth, S. Li et al., "Modelling and optimization of enhanced coalbed methane recovery using $\mathrm{CO}_{2} / \mathrm{N}_{2}$ mixtures," Fuel, vol. 253, pp. 1114-1129, 2019.

[4] A. S. Ranathunga, M. S. A. Perera, P. G. Ranjith, and C. H. Wei, "An experimental investigation of applicability of $\mathrm{CO}_{2}$ enhanced coal bed methane recovery to low rank coal," Fuel, vol. 189, pp. 391-399, 2017.

[5] K. Jin, Y. Cheng, T. Ren et al., "Experimental investigation on the formation and transport mechanism of outburst coal-gas flow: implications for the role of gas desorption in the development stage of outburst," International Journal of Coal Geology, vol. 194, pp. 45-58, 2018.

[6] J. Sobczyk, "A comparison of the influence of adsorbed gases on gas stresses leading to coal and gas outburst," Fuel, vol. 115, pp. 288-294, 2014.

[7] S. Xue, Y. Wang, J. Xie, and G. Wang, "A coupled approach to simulate initiation of outbursts of coal and gas-model 
development," International Journal of Coal Geology, vol. 86, no. 2-3, pp. 222-230, 2011.

[8] T. Lu, H. Yu, T. Zhou, J. Mao, and B. Guo, "Improvement of methane drainage in high gassy coal seam using waterjet technique," International Journal of Coal Geology, vol. 79, no. 1-2, pp. 40-48, 2009.

[9] L. Li, F. Wang, T. Li, X. Dai, X. Xing, and X. Yang, "The effects of inclined particle water jet on rock failure mechanism: experimental and numerical study," Journal of Petroleum Science and Engineering, vol. 185, article 106639, 2020.

[10] C. Cai, X. Wang, X. Yuan et al., "Experimental investigation on perforation of shale with ultra-high pressure abrasive water jet: shape, mechanism and sensitivity," Journal of Natural Gas Science and Engineering, vol. 67, pp. 196-213, 2019.

[11] S. Liu, F. Zhou, H. Li, Y. Chen, F. Wang, and C. Guo, "Experimental investigation of hard rock breaking using a conical pick assisted by abrasive water jet," Rock Mechanics and Rock Engineering, vol. 53, no. 9, pp. 4221-4230, 2020.

[12] Y. Xue, H. Si, D. Xu, and Z. Yang, "Experiments on the microscopic damage of coal induced by pure water jets and abrasive water jets," Powder Technology, vol. 332, pp. 139-149, 2018.

[13] D. Li, "A new technology for the drilling of long boreholes for gas drainage in a soft coal seam," Journal of Petroleum Science and Engineering, vol. 137, pp. 107-112, 2016.

[14] X. Li, D. Zhou, Y. Lu, Y. Kang, Y. Zhao, and X. Wang, "Dynamic effects of high-pressure pulsed water jet in lowpermeability coal seams," Journal of Coal Science \& Engineering, vol. 15, no. 3, pp. 284-288, 2009.

[15] S. Chen, T. Yang, P. G. Ranjith, and C. Wei, "Mechanism of the two-phase flow model for water and gas based on adsorption and desorption in fractured coal and rock," Rock Mechanics \& Rock Engineering, vol. 50, no. 3, pp. 571-586, 2017.

[16] J. Dong, Y. Cheng, K. Jin et al., "Effects of diffusion and suction negative pressure on coalbed methane extraction and a new measure to increase the methane utilization rate," Fuel, vol. 197, pp. 70-81, 2017.

[17] A. R. Shahani and M. Seyyedian, "Simulation of glass cutting with an impinging hot air jet," International Journal of Solids and Structures, vol. 41, no. 5-6, pp. 1313-1329, 2004.

[18] Y. Masip, A. Campo, and S. M. Nuñez, "Experimental analysis of the thermal performance on electronic cooling by a combination of cross-flow and an impinging air jet," Applied Thermal Engineering, vol. 167, p. 114779, 2020.

[19] Y. Lu, A. Klimenko, H. Russell, Y. Dai, J. Warner, and K. Hooman, "A conceptual study on air jet-induced swirling plume for performance improvement of natural draft cooling towers," Applied Energy, vol. 217, no. 18, pp. 496-508, 2018.

[20] T. Komori, M. Miura, S. Horiki, and M. Osakabe, "ICOPE-151158. Supersonic air and wet steam jet using simplified de Laval nozzle," in Proceedings of the International Conference on Power Engineering, Yokohama, Japan, 2015.

[21] A. Frendi and M. Brown, "Flow structures and noise from a supersonic impinging jet," International Journal of Numerical Methods for Heat and Fluid Flow, vol. 26, no. 8, pp. 2509-2527, 2016.

[22] H. Kutter and C. Fairhurst, "On the fracture process in blasting," International Journal of Rock Mechanics and Mining Sciences \& Geomechanics Abstracts, vol. 8, no. 3, pp. 181-202, 1971.

[23] T. N. Hagan, "Rock breakage by explosives," Acta Astronautica, vol. 6, no. 3-4, pp. 329-340, 1979.
[24] R. Nilson, W. Proffer, and R. Duff, "Modelling of gas-driven fractures induced by propellant combustion within a borehole," International Journal of Rock Mechanics and Mining Sciences \& Geomechanics Abstracts, vol. 22, no. 1, pp. 3-19, 1985.

[25] M. Eslamian, A. Pophali, M. Bussmann, and H. N. Tran, "Breakup of brittle deposits by supersonic air jet: the effects of varying jet and deposit characteristics," International Journal of Impact Engineering, vol. 36, no. 2, pp. 199-209, 2009.

[26] Y. Liu, H. Zhang, P. G. Ranjith, J. Wei, and X. Liu, "Wear mechanism of abrasive gas jet erosion on a rock and the effect of abrasive hardness on it," Geofluids, vol. 2019, Article ID 4125363, 14 pages, 2019.

[27] Y. Liu, T. Zhang, and X. Liu, "Analysis of the stress wave effect during coal breakage by a high-pressure abrasive air jet," Advances in Mechanical Engineering, vol. 10, no. 6, 2018.

[28] Y. Liu, J. Zhang, J. P. Wei, and X. T. Liu, "Optimum structure of a Laval nozzle for an abrasive air jet based on nozzle pressure ratio," Powder Technology, vol. 364, no. 3, pp. 343-362, 2020.

[29] Y. Liu, C. Wang, J. P. Wei, and C. Chen, "Effect of nozzle pressure ratio on pulsation frequency of air jets used in hole drilling," Journal of Petroleum Science and Engineering, vol. 196, article 107399, 2021.

[30] P. G. Ranjith, Y. Liu, J. Wei, and X. Liu, "Effect of abrasive mass flow on the abrasive acceleration and erosion rates of abrasive gas jets," Rock Mechanics and Rock Engineering, vol. 52, no. 9, pp. 3085-3102, 2019.

[31] X. Song, G. Wang, H. Ma, H. Gong, and C. Cui, "Mechanism and mathematical model of the continuous process of gas jet breaking coal," Journal of China Coal Society, vol. 45, no. 9, pp. 3176-3185, 2020.

[32] S. M. Simionescu, N. O. Tănase, D. Broboană, and C. Bălan, "Impinging air jets on flat surfaces at low Reynolds numbers," Energy Procedia, vol. 112, pp. 194-203, 2017.

[33] M. J. Tummers, J. Jacobse, and S. G. Voorbrood, "Turbulent flow in the near field of a round impinging jet," International Journal of Heat and Mass Transfer, vol. 54, no. 23-24, pp. 4939-4948, 2011.

[34] S. Beltaos and N. Rajaratnam, "Plane turbulent impinging jets," Journal of Hydraulic Research, vol. 11, no. 1, pp. 29-59, 1973.

[35] S. Beltaos and N. Rajaratnam, "Impingement of axisymmetric developing jets," Journal of Hydraulic Research, vol. 15, no. 4, pp. 311-326, 1977.

[36] J. G. A. Bitter, "A study of erosion phenomena part I," Wear, vol. 6, no. 1, pp. 5-21, 1963.

[37] A. V. Levy and W. Buqian, "Erosion of hard material coating systems,” Wear, vol. 121, no. 3, pp. 325-346, 1988.

[38] A. Kaliazine, M. Eslamian, and H. N. Tran, "On the failure of a brittle material by high velocity gas jet impact," International Journal of Impact Engineering, vol. 37, no. 2, pp. 131-140, 2010.

[39] S. Nakao and M. Takamoto, "Choking phenomena of sonic nozzles at low Reynolds numbers," Flow Measurement and Instrumentation, vol. 11, no. 4, pp. 285-291, 2000.

[40] S. Nakao, Y. Yokoi, and M. Takamoto, "Development of a calibration facility for small mass flow rates of gas and the uncertainty of a sonic Venturi transfer standard," Flow Measurement and Instrumentation, vol. 7, no. 2, pp. 77-83, 1996. 